\title{
Roughening kinetics of chemical vapor deposited copper films on $\mathrm{Si}(100)$
}

\author{
L. Vázquez and J. M. Albella
}

Instituto de Ciencia de Materiales (CSIC). 28049, Madrid, Spain

\author{
R. C. Salvarezza and A. J. Arvia \\ Instituto de Investigaciones Fisicoquímicas Teóricas y Aplicadas (CONICET), Sucursal 4, \\ Casilla de Correo 16, (1900) La Plata, Argentina \\ R. A. Levy and D. Perese \\ New Jersey Institute of Technology, University Heights, Newark, New Jersey 07102
}

(Received 25 September 1995; accepted for publication 19 December 1995)

\begin{abstract}
The roughening kinetics of copper films synthesized by low pressure chemical vapor deposition (LPCVD) on $\mathrm{Si}(100)$ substrates was investigated by scanning tunneling microscopy (STM). By applying the dynamic scaling theory to the STM images, a steady growth roughness exponent $\alpha=0.81 \pm 0.05$ and a dynamic growth roughness exponent $\beta=0.62 \pm 0.09$ were determined. The value of $\alpha$ is consistent with growth model predictions incorporating surface diffusion. The value of $\beta$, while higher than expected from these models, can be related to LPCVD processing conditions favoring growth instabilities. (C) 1996 American Institute of Physics. [S0003-6951(96)01409-8]
\end{abstract}

Aluminum films have commonly been used in the metallization of integrated circuit devices. However, as devices shrink further into the quarter-micron regime, an aluminumbased metallization would be unable to meet the low electrical resistivity and stringent electromigration resistance requirements. Copper is a suitable alternative choice because of its low bulk electrical resistivity (1.67 versus $2.65 \mu \Omega \mathrm{cm}$ for $\mathrm{Al}$ ) and its superior electromigration resistance which would allow higher current densities to be sustained on smaller linewidths as well as minimize the $R C$ delay time of the circuit. Compared to "line of sight" deposition techniques (such as sputtering and evaporation), low-pressure chemical vapor deposition (LPCVD) is an attractive processing method for achieving either selective or nonselective copper with excellent step coverage. Recent studies have focused on the use of (trimethylvinylsilyl) hexafluoroacetylacetonato copper I [(hfac) $\mathrm{Cu}(\mathrm{I}) \mathrm{TMSV}]$ as a precursor for the deposition of LPCVD $\mathrm{Cu}$ films at relatively low temperatures and high growth rates. Although studies on the growth kinetics and properties of such films have been reported, ${ }^{1}$ little is known about the evolution of the interface and its effects on subsequent film growth processes.

This paper focuses on the scanning tunneling microscopy (STM) imaging of $\mathrm{Cu}$ films grown by LPCVD from (hfac) $\mathrm{Cu}(\mathrm{I}) \mathrm{TMVS}$ on $\mathrm{Si}(100)$ wafers under nonequilibrium conditions and over a broad range of film thicknesses. The dynamic scaling ${ }^{2}$ analysis applied to STM images ${ }^{3}$ allows us to evaluate the dynamic and static roughness exponents, and to derive from them the contribution of different processes to the growth mechanism of LPCVD $\mathrm{Cu}$ films under nonequilibrium conditions.

The $\mathrm{Cu}$ films were synthesized in a SPECTRUM model 211, cold wall, single wafer, cassette-to-cassette, fully automated CVD reactor. Single crystal (100) silicon wafers, 10 $\mathrm{cm}$ in diameter, were taken directly from sealed containers and introduced with no further cleaning treatment into the reaction chamber. The root-mean-square, rms, surface rough-

a)Electronic mail: Jvara@emduam11.bitnet ness of the virgin wafers resulted to be $0.14 \mathrm{~nm}$ as measured by atomic force microscopy. The wafers were exposed on the back side (unpolished side) to a dual zone radiant heater. The temperature of the wafer was measured using a thermocouple.

The (hfac) $\mathrm{Cu}(\mathrm{I})$ TMVS liquid precursor used for the deposition of the $\mathrm{Cu}$ films was directly injected into the reaction chamber. Two vacuum pump stations were used for achieving the desirable pressures within the reaction chamber and for controlling the wafer transport system. The $\mathrm{Cu}$ films in this study were synthesized at a pressure of 100 mTorr, at a deposition temperature of $453 \mathrm{~K}$, and average growth rate of $0.5 \mathrm{~nm} \mathrm{~s} s^{-1}$. The values for the average $\mathrm{Cu}$ film thicknesses, $\langle h\rangle$, were determined through profilometry to be in the range of $44-2220 \mathrm{~nm}$.

The topography of $\mathrm{Cu}$ films was imaged with a Nanoscope III STM operating in air. STM measurements were made using a $0.25 \mathrm{~V}$ bias voltage with the tip (+) at $0.5 \mathrm{nA}$ constant current. Different Pt-Ir tips were used to discard occasional artifacts caused by the tip geometry itself. To minimize errors STM images with $512 \times 512$ pixels were taken. ${ }^{3}$ STM data were analyzed after fitting the instrument plane and applying a subtracting procedure. ${ }^{4}$

The rms roughness (interface width) $\xi$ for length $L$ and average thickness $\langle h\rangle$, for a system containing $N$ sites with single valued heights $h_{i}$, is defined by the expression

$$
\xi(l,\langle h\rangle)=\left[\frac{1}{N} \sum_{i=1}^{N}\left[h_{i}-\langle h\rangle\right]^{2}\right]^{1 / 2} .
$$

The dynamic scaling theory ${ }^{2}$ predicts that when $\langle h\rangle \rightarrow 0$, $\xi$ scales as

$$
\xi(\langle h\rangle) \propto\langle h\rangle^{\beta}
$$

whereas for $\langle h\rangle \rightarrow \infty$ it scales as

$$
\xi(L) \propto L^{\alpha},
$$

where $\beta$ and $\alpha$ are the dynamic and static surface roughness exponents, respectively. 


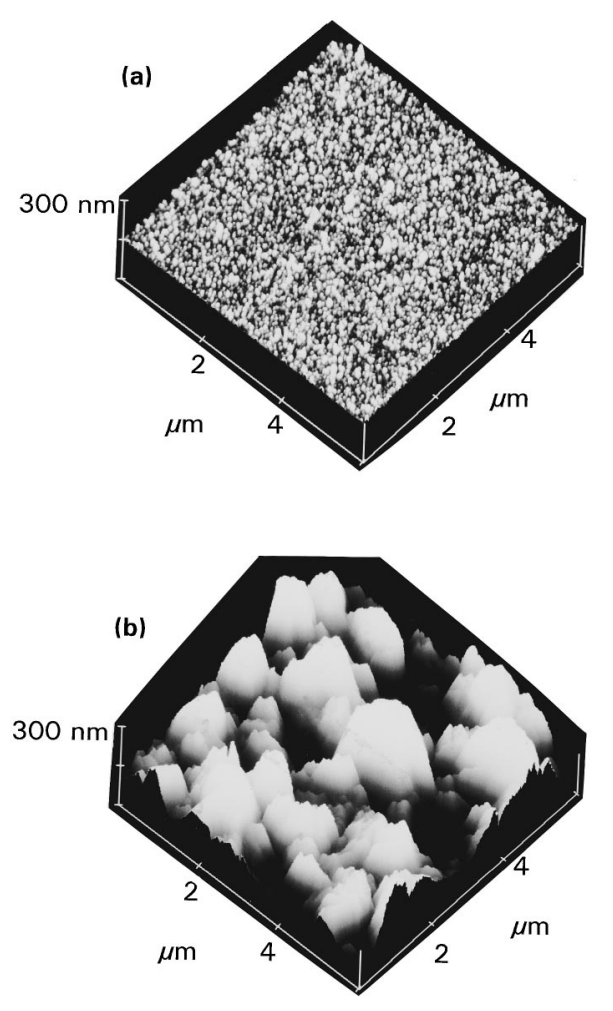

FIG. 1. Constant current $6 \times 6 \mu \mathrm{m}^{2}$ 3D STM images of CVD Cu films: (a) $\langle h\rangle=44 \mathrm{~nm}$; (b) $\langle h\rangle=2220 \mathrm{~nm}$. STM images have the same $z$ scale.

Atomistic-type growth models such as those of Eden, ${ }^{5}$ ballistic deposition, ${ }^{5}$ and restricted solid-on solid, ${ }^{5}$ have been proposed to explain nonequilibrium growth processes. These models can be successfully described by the Kardar, Parisi, and Zhang motion equation ${ }^{6}$ which leads to $\alpha=0.40$ and $\beta=0.25$ in $3 \mathrm{D}$ growth. Alternatively, continuous models in $3 \mathrm{D}$ incorporating surface diffusion yield either $\alpha=1.0$ and $\beta=0.25^{7,8}$ or $\alpha=0.66$ and $\beta=0.24$. $^{9}$

$\mathrm{Cu}$ film growth on $\mathrm{Si}(100)$ by the (hfac) $\mathrm{Cu}(\mathrm{I})$ TMVS procedure involves a progressive roughening for increasing values of $\langle h\rangle$ due to the competitive growth of $\mathrm{Cu}$ grains (Fig. 1). From Fig. 1(a) it is important to note that the $\mathrm{Cu}$ film seems to start to grow quite homogeneously. This homogeneity allows one to disregard effects due to improper cleanliness of the $\mathrm{Si}(100)$ substrate on the initial $\mathrm{Cu}$ film growth. It is known ${ }^{2}$ that as $\langle h\rangle$ increases the average grain size $d_{s}$ increases according to $d_{s} \propto\langle h\rangle^{\beta / \alpha}$. From the plot [Fig. 2(a)] of average film grain size (obtained from histograms of the grain size distributions) versus $\langle h\rangle$ a value for $\beta / \alpha=0.74$ is obtained. Also, for all values of $\langle h\rangle$, the experimental value of the interface width, $\xi_{\text {stm }}$, is seen to initially increase with $S$, the STM scan length, finally reaching a constant value $\xi_{\text {stm }}^{c}$ [Fig. 2(b)]. The value of $\xi_{\text {stm }}^{c}$ also increases with $\langle h\rangle$.

Accurate $\alpha$ values were obtained by using the single image dynamic scaling method. ${ }^{3}$ Accordingly, $\alpha$ was derived from the equation

$$
\xi_{\mathrm{stm}}(L) \propto L_{s}^{\alpha},
$$

where $L_{s}$ corresponds to a segment of the STM scan of size $S$ measured in the $x$ direction (i.e., the fast scanning direction) by plotting the $\log \xi_{\text {stm }}$ vs $\log L_{s}$ in the stationary
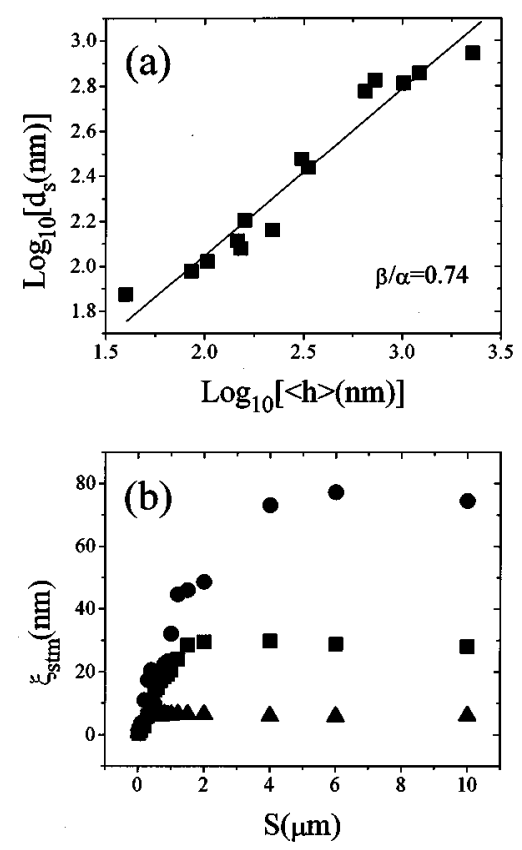

FIG. 2. (a) $\log _{10} d_{s}$ vs $\log _{10}\langle h\rangle$ plot, (b) $\xi_{\text {stm }}$ vs $S$ plot: $\langle h\rangle=44 \mathrm{~nm}(\mathbf{\Delta})$, $\langle h\rangle=1220 \mathrm{~nm}(\mathbf{\square})$ and $\langle h\rangle=2220 \mathrm{~nm}(\mathbf{\bullet})$.

roughness regime. For $\mathrm{Cu}$ deposits with $\langle h\rangle=44 \mathrm{~nm}$ and $\langle h\rangle=2220 \mathrm{~nm}$ [Fig. 3(a)] the $\log \xi_{\text {stm }}$ vs $\log L_{s}$ plots are seen to exhibit a linear region exceeding three decades in $\log L_{s}$, and a saturation region for $L_{s}$ values larger than the critical value $L_{\mathrm{sc}}$ which depends on $\langle h\rangle$. The value of $L_{\mathrm{sc}}$ at the crossover point corresponds closely to the value of $d_{s}$. From the slope of the straight lines depicted in Fig. 3, a value of $\alpha=0.82$ was determined. After averaging more than 300 different STM images, a value of $\langle\alpha\rangle=0.81 \pm 0.05$ was obtained. On the other hand, the exponent $\beta$ was evaluated from Eq. (2) (i.e., the variation of $\xi_{\text {stm }}^{c}$ vs $\langle h\rangle$ data). The
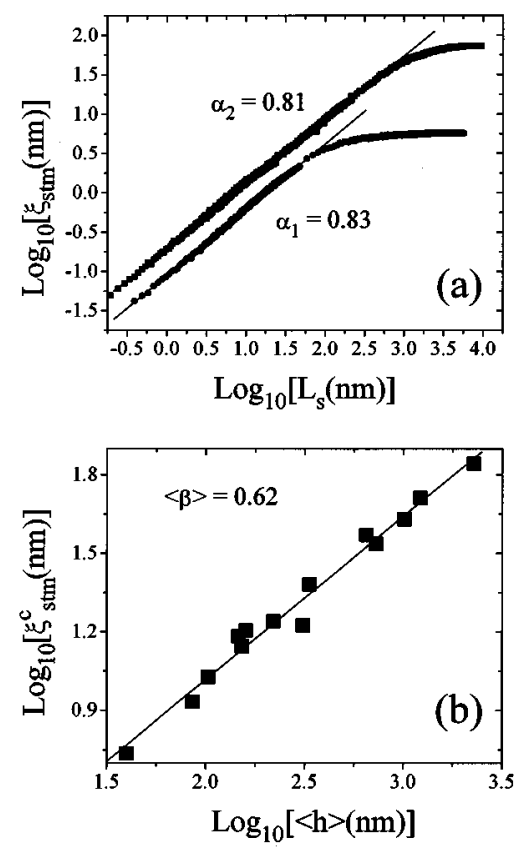

FIG. 3. (a) $\log _{10} \xi_{\text {stm }}$ vs $\log _{10} L_{s}$ plots for CVD Cu films: $\langle h\rangle=44 \mathrm{~nm}(\boldsymbol{\bullet}$, bottom curve), $\langle h\rangle=2220 \mathrm{~nm}$, (品, top curve). (b) $\log _{10} \xi_{\text {stm }}^{c} v^{2} \log _{10}\langle h\rangle$ plot. 
$\log \xi_{\text {stm }}^{c}$ vs $\log \langle h\rangle$ plot [Fig. 3(b)] resulting from 15 STM images for each value of $\langle h\rangle$ yielded a straight line with $\langle\beta\rangle=0.62 \pm 0.09$. These values yielded a ratio of $\beta / \alpha=0.77$, in agreement with the value derived from the $\log d_{s}$ vs $\log \langle h\rangle$ plot [Fig. 2(a)].

The $\alpha$ value obtained for LPCVD Cu films, 0.81 , is in agreement with other values $(0.9-0.7)$, reported for other experimental systems. They are consistent with the $\alpha$ values derived from growth models incorporating surface diffusion. Exponents $\alpha$ obtained from these models are in the $0.66-1$ range. ${ }^{7-9}$ Hence, in principle, surface diffusion appears to dominate roughness development for LPCVD $\mathrm{Cu}$ films grown at $T=453 \mathrm{~K}$. The preceding explanation is also consistent with recent data on the self-affine characteristics of vapor deposited $\mathrm{Au}$ grown in the range $298-673 \mathrm{~K}$ where surface diffusion was also found to dominate the growth process. ${ }^{10}$ It should be noted that both $\mathrm{Au}$ and $\mathrm{Cu}$ exhibit a large surface mobility even at room temperature. ${ }^{11}$

Nevertheless, the value $\beta=0.62$ is considerably higher than those derived from the growth models incorporating surface diffusion which predict either $\beta=0.25^{7,8}$ or $\beta=0.20{ }^{9}$ However, similar results have been reported for plasma polymer films, ${ }^{12}$ where the roughness exponents resulted in $\alpha=0.9$ and $\beta=0.7$, and for $\mathrm{Si}_{0.4} \mathrm{Ge}_{0.6}$ thin films grown by molecular beam epitaxy (MBE) on $\mathrm{Si}^{13}$ where an experimental $\beta$ value of 0.71 was interpreted as a result of instabilities in MBE growth. Also it has been recently suggested ${ }^{13}$ that the strain due to lattice mismatch in the heteroepitaxial films could be another source of growth instability.

For $\mathrm{Cu}$ films deposited by $\mathrm{MBE}$ on $\mathrm{Cu}(100)$ at $200 \mathrm{~K},{ }^{14}$ $\alpha \approx 1$ and $\beta=0.56$ were obtained. In this case the high $\beta$ value, close to that measured in this work, was explained as a consequence of the asymmetry in the vertical and horizontal mobility of adatoms (Schwöbel barriers) which tend to increase the value of $\beta$. For $\mathrm{Cu}$ electrodeposition the values $\alpha=0.9$ and $\beta=0.45$ were obtained. This high $\beta$ value was assigned to the influence of the electric field ${ }^{15}$ on the growth process. In general, experimental values of $\beta$ larger than those derived from growth models including local effects were observed when nonlocal effects arising from Laplacian fields were present during the interface growth. In LPCVD $\mathrm{Cu}$ film formation, it is possible that the pressure field created around the rough growing interface, presumably by TMVS produced in the rate determining step, ${ }^{1}$ results in the development of growth instabilities leading to a high value of $\beta$.

This work was partially supported by the CSICCONICET cooperation program and by a grant from the NASA New Jersey Space Grant Consortium.

${ }^{1}$ A. Jain, K. M. Chi, T. T. Kodas, and M. J. Hampden-Smith, J. Electrochem. Soc. 140, 1434 (1994).

${ }^{2}$ F. Family, Physica A 168A, 561 (1990).

${ }^{3}$ R. C. Salvarezza, L. Vázquez, P. Herrasti, P. Ocón, J. M. Vara, and A. J. Arvia, Europhys. Lett. 20, 727 (1992).

${ }^{4}$ J. Krim, I. Heyvaert, C. Haesendonck, and Y. Bruynseraede, Phys. Rev. Lett. 70, 57 (1993).

${ }^{5}$ A.-L. Barabási and H. E. Stanley, Fractal Concepts in Surface Growth (Cambridge University Press, Cambridge, England, 1995).

${ }^{6}$ M. Kardar, G. Parisi, and Y. C. Zhang, Phys. Rev. Lett. 56, 889 (1986).

${ }^{7}$ J. Villain, J. Phys. I 1, 19 (1992).

${ }^{8}$ M. Siegert and M. Plischke, Phys. Rev. Lett. 73, 1517 (1994).

${ }^{9}$ Z. W. Lai and S. Das Sarma, Phys. Rev. Lett. 66, 2348 (1991).

${ }^{10}$ L. Zubimendi, M. E. Vela, R. C. Salvarezza, L. Vázquez, J. M. Vara, and A. J. Arvia, Phys. Rev. E 50, 1367 (1994).

${ }^{11}$ H. P. Bonzel, in Surface Physics of Materials, edited by J. M. Blakely (Academic, New York, 1975), p. 280.

${ }^{12}$ G. W. Collins, S. E. Letts, E. M. Fearon, R. L. McEachern, and T. P. Bernat, Phys. Rev. Lett. 73, 708 (1994).

${ }^{13}$ Z. H. Ming, S. Huang, Y. L. Soo, Y. H. Kao, T. Carns, and K. L. Wang, Appl. Phys. Lett. 67, 629 (1995).

${ }^{14}$ H.-J. Ernst, F. Fabre, R. Folkerts, and J. Lapujoulade, Phys. Rev. Lett. 72, 112 (1994).

${ }^{15}$ A. Iwamoto, T. Yoshinobu, and H. Iwasaki, Phys. Rev. Lett. 72, 4025 (1994). 